Research article

\title{
Characterization of BTBDI and BTBD2, two similar BTB-domain-containing Kelch-like proteins that interact with Topoisomerase I Lixin $\mathrm{Xu} \dagger^{1}$, Lihong Yang $\dagger^{1,2}$, Keiko Hashimoto ${ }^{1}$, Melvin Anderson ${ }^{1}$, Glenda Kohlhagen ${ }^{3}$, Yves Pommier ${ }^{3}$ and Peter D'Arpa*1
}

Address: ${ }^{1}$ Department of Biochemistry and Molecular Biology Uniformed Services University of the Health Sciences 4301 Jones Bridge Road, Bethesda, MD 20814-4799, USA, ${ }^{2}$ Laboratory of Cellular and Molecular Biology, National Cancer Institute, Bldg. 37/Rm. 1C18, Bethesda, MD 20892-4255, USA and ${ }^{3}$ Laboratory of Molecular Pharmacology, Center for Cancer Research, National Cancer Institute, National Institutes of Health, Bethesda, MD 20892-4255, USA

E-mail: LixinXu - lixu@usuhs.mil; Lihong Yang - yanglih@mail.nih.gov; Keiko Hashimoto - khashimoto@usuhs.mil; Melvin Anderson - mander11@gl.umbc.edu; Glenda Kohlhagen - kohlhagg@mail.nih.gov; Yves Pommier - pommiery@pop.nci.nih.gov; Peter D'Arpa* - pdarpa@usuhs.mil

*Corresponding author †Equal contributors

Published: 7 January 2002

BMC Genomics 2002, 3:I
Received: 17 October 2001

Accepted: 7 January 2002

This article is available from: http://www.biomedcentral.com/I47I-2/64/3/I

(C) 2002 Xu et al; licensee BioMed Central Ltd. Verbatim copying and redistribution of this article are permitted in any medium for any non-commercial purpose, provided this notice is preserved along with the article's original URL. For commercial use, contact info@biomedcentral.com

\begin{abstract}
Background: Two-hybrid screening for proteins that interact with the core domain of human topoisomerase I identified two novel proteins, BTBDI and BTBD2, which share $80 \%$ amino acid identities.

Results: The interactions were confirmed by co-precipitation assays demonstrating the physical interaction of BTBDI and BTBD2 with $100 \mathrm{kDa}$ topoisomerase I from HeLa cells. Deletion mapping using two-hybrid and GST-pulldown assays demonstrated that less than the C-terminal half of BTBDI is sufficient for binding topoisomerase I. The topoisomerase I sequences sufficient to bind BTBD2 were mapped to residues 215 to 329 . BTBD2 with an epitope tag localized to cytoplasmic bodies. Using truncated versions that direct BTBD2 and TOPI to the same cellular compartment, either the nucleus or the cytoplasm, co-localization was demonstrated in cotransfected Hela cells. The supercoil relaxation and DNA cleavage activities of topoisomerase I in vitro were affected little or none by co-incubation with BTBD2. Northern analysis revealed only a single sized mRNA for each BTBDI and BTBD2 in all human tissues tested. Characterization of BTBD2 mRNA revealed a 255 nucleotide $90 \%$ GC-rich region predicted to encode the $\mathrm{N}$-terminus. $B T B D /$ and $B T B D 2$ are widely if not ubiquitously expressed in human tissues, and have two paralogs as well as putative orthologs in $C$. elegans and D. melanogaster.
\end{abstract}

Conclusions: BTBDI and BTBD2 belong to a small family of uncharacterized proteins that appear to be specific to animals. Epitope-tagged BTBD2 localized to cytoplasmic bodies. The characterization of BTBDI and BTBD2 and their interaction with TOPI is underway.

\section{Background}

DNA topoisomerase I (TOP1) is a ubiquitously expressed protein that relaxes DNA supercoils generated during many transactions of DNA including replication, transcription, repair, and chromatin condensation and remodeling. TOP1 has been shown to interact with many 
proteins including components of the holo TFIIIC complex [1], TFIID [2], p53 [3], SV-40 large T antigen [4], nucleolin [5], the RING finger protein topors [6], the RNA splicing factors PSF/p54 ${ }^{\text {nrb [7] }}$ and SF2/ASF [8], the cell cycle regulatory protein ARF [9], RNA pol II complex $[10,11]$, and HTLV type 1 tax oncoprotein [12]. TOP1 also interacts with a variety of unidentified proteins involved in its phosphorylation, ubiquitination and sumoylation [13-17].

We used the core domain of TOP1 lacking the N-terminal highly charged region, the coiled-coil linker domain and the C-terminal active site domain as bait in two hybrid screens for TOP1 interacting proteins. We obtained multiple independent cDNA clones coding for BTBD1 and BTBD2, two uncharacterized, BTB domain-containing, kelch-like proteins. The interactions of HeLa cell TOP1 with the BTBD proteins was confirmed using GST-pulldown and was further suggested by their intracellular colocalization.

\section{Results \\ Identification of TOPI-interacting proteins using two-hy- brid assays}

The core domain of human TOP1 from residue 198 to 651 was used as the bait in two-hybrid screens [18-20]. This region of TOP1 lacks the $\mathrm{N}$-terminal $\sim 25 \%$ of the protein that is highly charged and the C-terminal domain containing the active site and the coiled-coil linker. The resulting polypeptide comprises $60 \%$ of the TOP 1 sequence. A screen of a HeLa cell library yielded 56 colonies that tested positive in the two hybrid assays (growth on plates lacking histidine or uracil, lack of growth on plates containing 5fluoroorotic acid; and induction of lacZ resulting in blue color when assayed with X-gal). Nucleotide sequence analysis of 34 of these clones revealed two cDNA contigs of 15 and 19 that predict proteins whose sequences are $80 \%$ identical. The proteins were named BTBD1 and BTBD2 according to a recent analysis of their genomic sequences [21]. The clones of each contig differed in the amount of $5^{\prime}$ sequence present: $11 / 15$ and $15 / 19$ of BTBD1 and BTBD2, respectively, had different 5' ends. In the two-hybrid growth assays and $\beta$-galactosidase assays, each of the clones tested as positive as the control for strongly interacting proteins (the interaction of c-Fos with c-Jun, ProQuest, Life Technologies). Another two-hybrid screen using a different two-hybrid system (Matchmaker, Clontech) also yielded 2 clones identical to the contig of 15 clones (BTBD1).

\section{Characterization of BTBDI and BTBD2 mRNA}

The longest BTBD1 cDNA clone obtained from two-hybrid screening (3196 bp) contains an ORF that encodes a 482 aa protein starting with an ATG that is preceded by an in-frame stop codon. Sequences in GenBank confirmed our sequence. One was obtained by the oligo-capping method (FLJ20724), and another assembled from partial cDNA and RACE fragments [21].

The longest BTBD2 cDNA obtained from two-hybrid screening (2413 bp) contained an ORF starting at the 5' end. To obtain the missing 5' cDNA, a nested PCR strategy was used to amplify the sequence from four independent cDNA libraries, but no extended sequences were obtained. 5'-RACE (Life Technologies) also failed to produce longer clones. This difficulty to obtain longer cDNA clones was seen by others [21]. The GC richness at the 5 ' end (85 nucleotides are $79 \%$ GC) may form secondary structures that block reverse transcriptase. Analysis of the BTBD2 gene immediately upstream of the 5 ' end of our cDNA predicts an ORF that begins with an ATG and encodes 85 amino acids in-frame with the ORF of our longest BTBD2 clone; however, the 255 nucleotides encoding these in-frame amino acids sequence are $89 \%$ GC rich. To determine if this sequence might be part of the BTBD2 mRNA, RT-PCR followed by nested PCR was used. Two antisense primers complementary to exon 2 were used to prime reverse transcription. Sense primers for PCR amplification were located within predicted exon 1 (Fig. 1A). Amplified products of 460 and $306 \mathrm{bp}$ that are predicted from the mRNA template resulted (Fig. 1B). If genomic DNA had been the template, products greater than $18 \mathrm{~kb}$ would have been obtained. Furthermore, sequencing of the 460 bp-amplification product confirmed it to be the predicted mRNA (Fig. 1A). The cDNA starting at the predicted start codon was deposited in GenBank (AF355797).

\section{Northern blotting and in silico expression analysis}

Northern blotting was used to determine the tissue distribution and sizes of the mRNA encoding BTBD1 and BTBD2 (Fig. 2). Poly (A)+RNA from human tissues $(2 \mu \mathrm{g} /$ lane) was probed with BTBD1, BTBD2 or TOP1 cDNA. Each mRNA was detected in all the tissues tested. BTBD1 mRNA was highly expressed in testes, heart and skeletal muscle. BTBD2 was more highly expressed in skeletal muscle. Only a single message size was detected for each BTBD1 (3.2 kb) and BTBD2 (2.8 kb).

BTBD1 and BTBD2 cDNA were found in libraries from the following tissues: amniotic, aorta, bone, brain, bone marrow, colon, ear, embryo, foreskin, gall bladder, germ, heart, kidney, lung, muscle, ovary, pancreas, parathyroid, placenta, skin, spleen, stomach, testis, thymus, tonsil and uterus (LocusID 53339 and 55643, respectively [22]). BTBD1 and BTBD2 was also expressed in a large number of tumor types and normal tissues present in SAGE (serial analysis of gene expression) libraries (SAGEmap, [23]). 
A

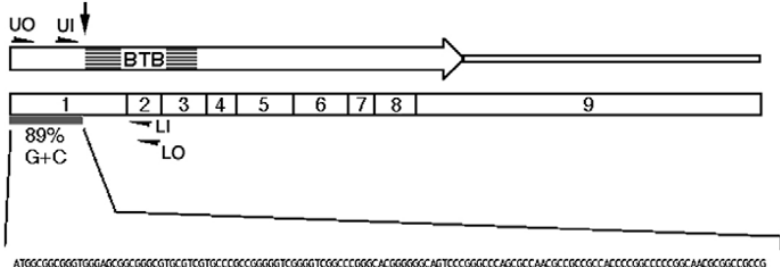

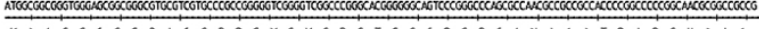

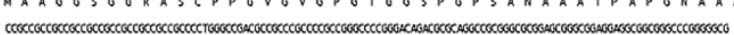
A A A A A A A A A A A P OPTPPAPPGPGTOAQAAGAERAEEAAGPGA

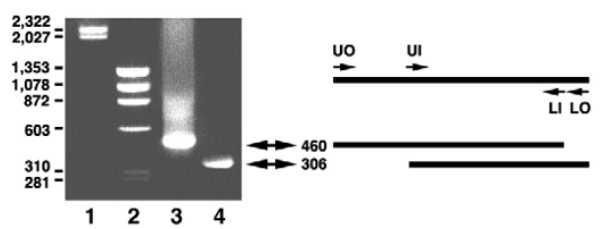

Figure I

RT-PCR identifies a highly GC-rich sequence predicted to encode the ammo terminal region of BTBD2. A. RT-PCR strategy and the sequence of the amplified region are shown: BTBD2 coding region (open arrow), 3'-UTR (narrow open bar) and exons (wide open bar, I - 9), sense primers (UO and $\mathrm{UI}$ ), antisense primers (LI and LO), GC-rich region (solid bar and sequence). B. Products of the RT-PCR are shown on an ethidium bromide gel. Reverse transcription was performed with antisense primers complementary to exon 2. The schematic shows the relevant portion of the mRNA template, the RT primers ( $\mathrm{LI}$ and LO), the sense primers (UO and UI), and the amplified products (460 and 306 bp) that correspond to the bands on the agarose gel stained with ethidium bromide (lanes I \& 2, DNA size standards; lane 3, product of reverse transcription with $L O$, Ist round $P C R$ with $U O$ and $L O$, and $2^{\text {nd }}$ round PCR with $U O$ and $L I$; lane 4 , product of reverse transcription with $L O, I^{\text {st }}$ round $P C R$ with $U O$ and LO, and $2^{\text {nd }}$ round PCR with $U I$ and LO.

\section{Mapping of the regions of TOPI and the BTBDs that inter- act}

BTBD1 and BTBD2 were mapped by deletion analysis to identify amino acid sequences required for interaction with TOP1. In two-hybrid assays, N-terminal deletions of $\sim 36 \%$ of BTBD1 (177 aa) or 43\% BTBD2 (228 aa) did not eliminate binding to TOP1 (Fig. 3A+B). In contrast, C-terminal deletions of 112 and 33 aa of BTBD1 and BTBD2, respectively, eliminated binding to TOP1 in the two-hybrid assays. Thus sequences very close to the C-terminus are required for the interaction with TOP1 by this analysis.

Two-hybrid analysis was also used to map regions of the TOP1 core domain that interact with BTBD2. The region upstream of aa 215 and downstream of aa 329 were not required for binding BTBD2 (Fig. 3C). The fragment of TOP1 from amino acid 215 to 331 was capable of binding to BTBD2. This region includes 18 aa of core subdomain
I, all of core subdomain II and 10 amino acids of the second section of core subdomain I.

\section{GST-pulldown assays confirm two-hybrid deletion map- ping}

Truncated fragments of BTBD1 and BTBD2 were tested in GST-pull down assays for their ability to bind TOP1 in nuclear extracts of HeLa cells. The GST-pulldown assay confirmed the positive interactions seen in the two-hybrid deletion mapping (Fig. 4). In addition, we found that two $\mathrm{N}$-terminal truncated BTBD1 and BTBD2 were able to coprecipitate TOP1 from nuclear extracts (BTBD1, 213-482 \& 303-482; BTBD2, 249-525 \& 289-525) although they were negative in the two-hybrid assay. Since truncated fragments may be unstable in yeast and may not accumulate to levels sufficient to produce a positive result, we used roughly equal amounts of each truncated protein to test for binding to TOP1 in nuclear extracts. Taken together, the results of the two-hybrid and GST-pulldown assays narrow the interacting region of BTBD1 and BTBD2 to roughly the C-terminal half of each protein with critical residues within the last 50 residues.

\section{Effect of GST-BTBD2 on TOPI activity}

To test the effect of BTBD2 on the supercoil relaxation activity of TOP1, HeLa cell nuclear extracts containing TOP1 were incubated with GST or GST-BTBD2 (aa 86-525). GST-BTBD2 slightly inhibited the supercoil relaxation activity of TOP1, increasing the time required to completely relax the supercoiled DNA by about two-fold as compared with GST alone (Fig. 5A). The DNA cleavage assay was also used to determine if BTBD2 affected TOP1 activity. This assay measures the ability of TOP1 to cleave end-labeled DNA substrates in the presence of the anticancer drug camptothecin, which blocks the ligation step of the cleavage/religation reaction of TOP1. A 36 bp DNA containing a single strong TOP1 cleavage site [24] or a $161 \mathrm{bp}$ restriction fragment [25] was used as the substrate. GSTBTBD2 addition produced a slight inhibition of TOP1 cleavage activity relative to GST alone (Fig. 5B) on the smaller substrate and had no detectable effect on cleavage activity or cleavage site selection by TOP1 on the larger substrate (5C).

\section{Cellular localization of BTBD2 and co-localization with TOPI}

To determine the localization of BTBD2 in cells, we transiently transfected HeLa cells with expression plasmids encoding either myc-tagged full length BTBD2 (mBTBD2F) or a truncated BTBD2 having the N-terminal half of the BTB domain removed (m-BTBD2T). The full length BTBD2 localized entirely to cytoplasmic bodies (Fig. 6B, m-BTBD2F). The truncated BTBD2 localized predominantly to nuclear bodies with a minor fraction localizing to cytoplasmic bodies (Fig. 6C, m-BTBD2T). 


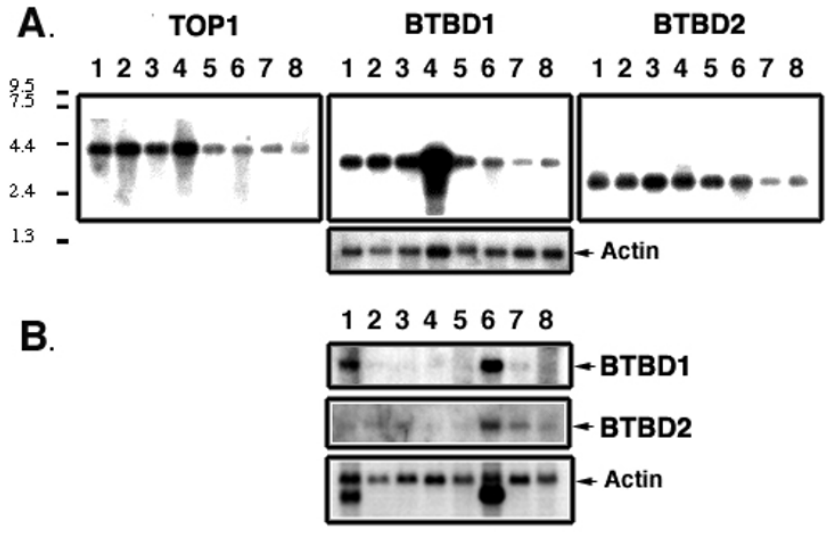

Figure 2

BTBDI and BTBD2 Northern blots of human tissues. Multiple Tissue Northern (MNT ${ }^{\mathrm{TM}}$, Clontech) blots were probed with 32P-dCTP-labeled cDNA (TOPI, BTBDI or BTBD2). Each lane contains $2 \mu \mathrm{g}$ of polyA+RNA from each tissue. A. I. spleen, 2. thymus, 3. prostate, 4. testes, 5. uterus, 6. small intestine, 7. colon, 8. leukocytes. B. I. heart, 2. brain, 3. placenta, 4. lung, 5. liver, 6. skeletal muscle, 7. kidney, 8. placenta. The membranes were re-probed for actin.

The localization of full length BTBD2 to cytoplasmic bodies was surprising because the cytoplasm is separated from the nucleus where endogenous TOP1 resides and transfected GFP-TOP1 localizes [26-30]. Because TOP1 and BTBD2 are not predominantly found in the same compartments, they may interact transiently, or they may interact only under specific conditions when they may be in the same compartment. We therefore analyzed mitotic cells, but did not observe co-localization of TOP1 and the BTBDs (not shown). We then tested for co-localization in transfected cells. Though the truncated m-BTBD2T localized to distinct nuclear bodies, its co-localization with endogenous TOP1 could not be determined against the background of endogenous TOP1 diffuse nuclear staining. However, in cells co-transfected with GFP-TOP1 and $\mathrm{m}$-BTBD2T, the $\mathrm{m}$-BTBD2T staining was nuclear diffuse as well as in nuclear bodies, indicating that $\mathrm{m}-\mathrm{BTBD} 2 \mathrm{~T}$ interacts with GFP-TOP1, which localizes throughout the nucleus (not shown).

To determine if TOP1 co-localizes with BTBD2 in the cytoplasm, GFP-TOP1 lacking an NLS [30] was co-transfected with full length BTBD2 (m-BTBD2F) or truncated BTBD2 (m-BTBD2T). GFP-TOP1 lacking a NLS (GFP-pTI6) localizes diffusely within the cytoplasm (Fig. 6A and [30]). In cells transfected with both GFP-pTI-6 and full length m-BTBD2F, the diffuse cytoplasmic GFP-pTI-6 significantly decreased, and GFP-pTI-6 co-localized with mBTBD2F to cytoplasmic bodies (Fig. 6D). In cells transfected with GFP-pTI-6 and truncated m-BTBD2T, truncated
m-BTBD2T was not in nuclear bodies as when transfected alone, rather it co-localized with GFP-pTI-6 to cytoplasmic bodies (Fig. 6E). Thus, when co-transfected with GFPTOP1 lacking a NLS, both full length and the N-terminal deleted BTBD2 co-localized with TOP1 to cytoplasmic bodies.

\section{Discussion}

Two-hybrid screens have identified BTBD1 and BTBD2 as proteins that interact with the core domain of TOP1. BTBD1 and BTBD2 were isolated as 15 and 19 independent clones, respectively, and in two-hybrid assays they interacted as strongly as the control for strongly interacting proteins (c-Fos with c-Jun). BTBD1 and BTBD2 also bound HeLa cell TOP1 (100 kDa) in nuclear extracts by GST-pulldown assays. N-terminal truncation studies showed the C-terminal $37 \%$ to $45 \%$ of the BTBD proteins are sufficient for binding TOP1. C-terminal deletions of either protein eliminated binding to TOP1 (C-terminal truncation of 112 aa of BTBD1 or 33 aa of BTBD2 in the two-hybrid assay; or a C-terminal truncation of 40 aa of BTBD2 in GST-pulldown). Truncation of the BTB domains demonstrated that they were not required for interaction with TOP1. The region of TOP1 sufficient for

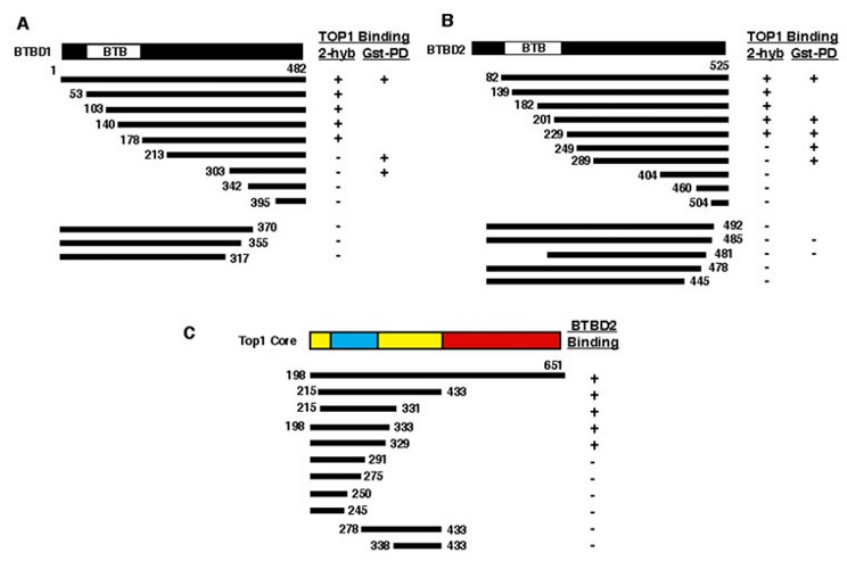

Figure 3

Deletion mapping of the interacting regions of TOPI and BTBDI and BTBD2 using two-hybrid assays.A. Deletions of BTBDI and their interaction with CTOP I (residues 19865I). B. Deletions of BTBD2 and their interaction with cTOPI. C. Deletions of CTOPI and their interaction with BTBD2. Interactions were tested using colony formation assays on plates lacking histidine or uridine and the $\beta$-galactosidase colony color assay. The TOPI core domain regions are shown from $\mathrm{N}$ - to C-terminus: subdomain I (yellow), subdomain II (blue), subdomain I (yellow) and subdomain III (red). The shortest clones obtained from two-hybrid screening were downstream of the BTB domains, beginng at residue 186 and 219 for BTBDI and BTBD2, respectively. Results are summarized for the two-hybrid (2-hyb) and the GST-pull down assays (Gst-PD, see Fig. 4) 
binding BTBD2 was narrowed to residues 215 - 329 in the "cap" region (core subdomain II) [31]. Catalytic activities of TOP1 were affected slightly or not at all by co-incubation with GST-BTBD2 suggesting that the interaction does not regulate enzyme activity directly.

We cloned the full length BTBD1 cDNA from a HeLa cell library. This sequence is $3196 \mathrm{bp}$, and the start codon is preceded by a stop codon 6 nucleotides upstream (GenBank, FLJ20724). Our sequence is comparable to two others in GenBank, one of which was obtained by the oligocapping method suggesting that it begins at the $5^{\prime}$ cap $[21,32]$.

Our repeated attempts to clone the 5' end of the BTBD2 by library screening and the RACE method were unsuccessful. We tested sequences directly upstream of the longest CDNA in the BTBD2 gene for their presence on the mRNA. RT-PCR and sequencing of the RT-PCR product demonstrated that this 255 nucleotide, $89 \%$ GC-rich sequence is present on the BTBD2 mRNA and that it shares predicted

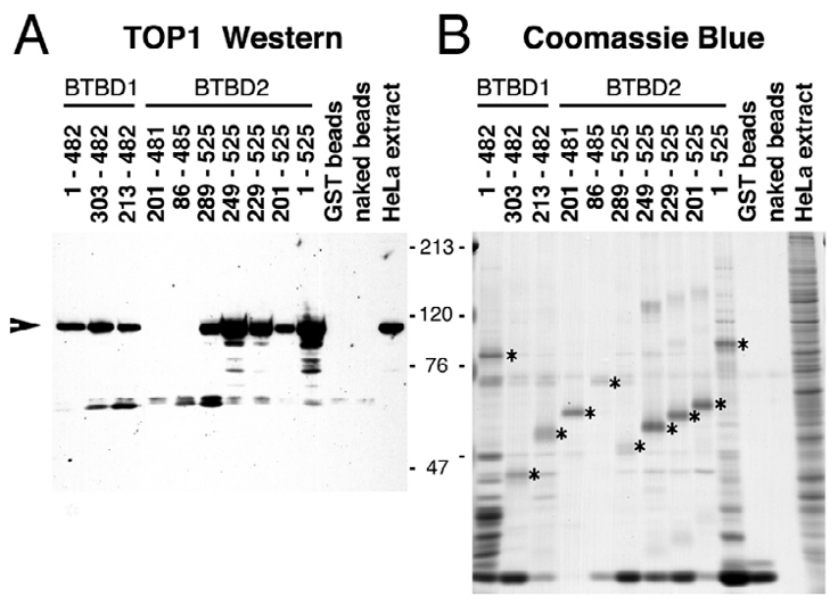

\section{Figure 4}

TOPI from HeLa cells interacts with GST-BTBDI and GSTBTBD2.A.TOPI Western blot. HeLa cell nuclear extracts were incubated with GST or GST-BTBD2 beads. After washing, the beads were solubilized in SDS-PAGE sample buffer and subjected to SDS-PAGE and Western blotting with TOPI antiserum. B.Coomassie Blue stained gel showing the quantity of GST and GST fusion proteins added to the binding reactions (*). METHODS: GST-BTBDI or GST-BTBD2 was expressed and purified from $E$. coli extracts. $\sim 5 \mu \mathrm{g}$ of GST or the GST-fusion proteins were mixed with HeLa cell nuclear extracts and incubated for $2 \mathrm{hr}$ at $4^{\circ} \mathrm{C}(0.25 \mathrm{M} \mathrm{NaCl})$. The bead pellets were then washed 3 times with ice cold PBS before solubilization in SDS sample buffer. One tenth of the nuclear extract used in the binding reaction was loaded in the lanes labeled "HeLa extract". Arrow shows co-precipitated TOPI. polypeptide sequence with BTBD homologues and orthologs.

The BTBD proteins contain an amino terminal BTB/POZ domain as well as a $\mathrm{C}$-terminal region that is related to proteins that contain the kelch domain. Neither of these regions yields unequivocal information for functional classification. The BTB domain is a highly conserved 120 amino acid, hydrophobic-rich, protein-protein interaction domain that mediates homodimerization and heterodimerization of a large number of proteins with diverse functions [9]. The three dimensional structure of one BTB domain [33] has been characterized through structure-function studies [34]. Proteins containing BTB domains are currently categorized into three groups [35]: 1) DNA-binding proteins often containing zinc fingers in the C-terminal region, 2) actin binding proteins that contain a kelch repeat of $\sim 50$ residues that terminate in a pair of glycine residues [36] and 3) proteins that have neither an actin-binding domain nor a DNA-binding domain. The BTBD proteins belong to the last category, although they are distantly related to kelch proteins ( $\sim 25 \%$ identical over $\sim 250 \mathrm{aa}$ ), they lack the residues that are characteristic of kelch repeats, a double glycine sequence and a tyrosine separated from a tryptophan by precisely 6 residues $[35,37]$.

BTBD1 and BTBD2 are closely related sharing 80\% identical amino acid sequence. They are one of eleven genes in 19 p13.3 $\rightarrow$ p12 with close homology to genes in 15 q $24 \rightarrow$ q26 that have apparently resulted from duplications of a common ancestral gene [38]. Both BTBD1 and BTBD2 are widely if not ubiquitously expressed in human tissues. The predicted promoter regions are highly GC-rich and lack canonical TATA boxes, suggesting that they may be housekeeping genes $[39,40]$.

Close mammalian relatives of BTBD1 and BTBD2 include the mouse orthologs that are 98\% and 95\% identical respectively, as well as highly similar rat and bovine partial sequences [21]. Sequence relatives in lower organisms include a $C$. elegans sequence with $58 \%$ identities with BTBD2 (429 residues; 2\% gaps, F38H4.7), and a D. melanogaster sequence with $46 \%$ identities (437 residues, 3\% gaps, CG5319). The human genome encodes two proteins with 46\% (436 residues, 3\% gaps, CAC22147) and 45\% (497 residues, 5\% gaps, AF353674) identities compared with BTBD2. These proteins comprise a small family whose members have not been functionally characterized and are evidently specific to animals because putative orthologs are not present in available fungi and plant sequence databases.

We have demonstrated an interaction between BTBD2 and TOP1 by two-hybrid, GST-pull down and intracellu- 
A

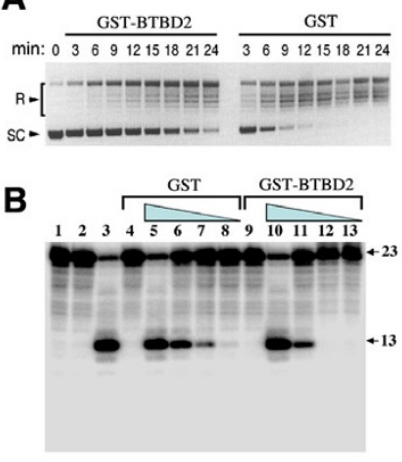

C

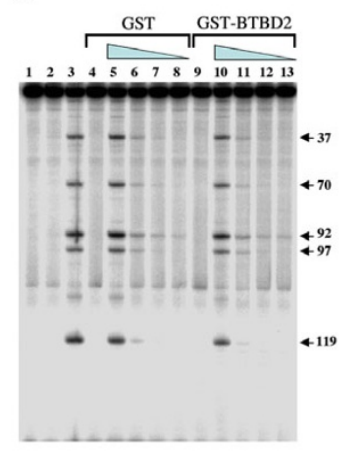

Figure 5

Effect of BTBD2 on TOPI activity in vitro.A.Supercoil relaxation activity. HeLa cell nuclear extract containing TOPI was mixed with GST-BTBD2 or GST alone for 15 min on ice before the addition of supercoiled DNA. The reactions were then incubated for $0-24 \mathrm{~min}$ at $30^{\circ} \mathrm{C}$ before stopping with SDS. The location of relaxed and supercoiled topoisomers are indicated (R and SC). B.DNA nicking at a strong TOPI cleavage site. GST or GST-BTBD2 was incubated with TOPI for $20 \mathrm{~min}$ at room temperature before the addition of a 36 bp 3' end-labeled DNA [24]. Cleavage reactions were continued for another 20 min before stopping with SDS. Lane I: DNA alone; lane 2: + TOPI (I.2 ng/reaction); lane 3: same + $10 \mu \mathrm{M}$ camptothecin; lanes 4-8: reactions contained $0.25 \mu \mathrm{g}$ GST and $10 \mu \mathrm{M}$ camptothecin; lanes 9-13 contained $0.25 \mu \mathrm{g}$ GST-BTBD2 + $10 \mu \mathrm{M}$ camptothecin; lanes 4 \& 9: no TOPI added; lanes 5 \& I0: I.2 ng TOPI; lanes 6 \& II: 0.4 ng TOPI; lanes 7 \& 12: $0.3 \mathrm{ng}$ TOPI; lanes 8 \& 13: $0.1 \mathrm{ng}$ TOPI). C. As in $B$, but using a $16 \mathrm{I}$ bp substrate DNA [25]. Numbering of cleavage sites according to Pommier et al. [25] is indicated on the right side.

lar co-localization analyses. Studies are underway to functionally characterize BTBD1 and BTBD2 and understand their interaction with TOP1.

\section{Conclusions}

- Fifteen and nineteen independent cDNA clones of BTBD1 and BTBD2, respectively, were isolated from twohybrid screens and found to interact as strongly as c-Fos interacted with c-Jun in the two-hybrid assays.

- The interactions of TOP 1 with BTBD1 and BTBD2 were confirmed by deletion mapping using two-hybrid and GST-pulldown assays.

- Less than the C-terminal half of each BTBD protein lacking the BTB domain is sufficient for binding to TOP1. TOP1 from aa 215 to 329 interacted with BTBD2.

- GFP-BTBD2 localized to cytoplasmic bodies.
- BTBD2 had little effect on the supercoil relaxation or DNA cleavage activity of TOP1 in vitro.

- Co-transfected BTBD2 and TOP1 co-localized.

- The BTBD2 message contains 255 nucleotides that are $89 \%$ GC-rich and are predicted to encode the N-terminus of a 525 amino acid protein.

- BTBD1 and BTBD2 mRNA were detected in all human tissues tested. BTBD1 mRNA was highly expressed in testes, heart and skeletal muscle. BTBD2 was more highly expressed in skeletal muscle. Only a single mRNA species was present for each BTBD1 and BTBD2.

- Putative orthologs of BTBD1 and BTBD2 are found in $C$. elegans (58\% identities, 429 residues; 2\% gaps, F38H4.7) and D. melanogaster (46\% identities, 437 residues, 3\% gaps, CG5319).

- Two paralogs of BTBD1 and BTBD2 are present in the human genome (CAC22147 and AF353674).

\section{Materials and Methods Plasmid construction}

To construct the bait expression plasmid (pGBD-cTOP1), the core domain of TOP1 (cTOP1, amino acids 198-651) was amplified by PCR (sense primer: 5'-CGGAATTCGAAGAGGAACAGAAGTGGAAATGG-3'; antisense primer: 5'CGGGATCCTTAAATCTTAGTTTGCAAGTTCATCAT-3')

and inserted into the vector pGBD (Clontech) cut with EcoRI and BamHI. To construct the cTOP1 expression vector (pDBleu-TOP1c), cTOP1 was excised from pGBDcTOP1 with EcoRI and BamHI and inserted into the same sites in pDBleu (ProQuest, Life Technologies).

To construct the GST-fusion protein expression vectors, the BTBD inserts from the prey vector pPC86 were amplified using primers complementary to the BTBD coding regions with additional Sma I and EcoR I restriction sites for insertion into these sites in pGEX-2TK (Pharmacia). These primer sequences can be found below under the Two $\mathrm{Hy}$ brid Screens section. All constructs were confirmed by sequencing using pGEX sequencing primers (Pharmacia Cat\# 27-1410-01 and 27-1411-01).

To construct the expression plasmid encoding C-terminalmyc-tagged, full length BTBD2 (m-BTBD2F), the entire coding region of BTBD2 (1-1575 bp) was amplified with primers (Upper primer: 5' ATGGCGGCGGGTGGGAGCGGCGGGCGTGCGTCGTG-3', Lower primer: 5'-GGTGTAGAAGATGACCTCGGGGATCTGGCCGTCCTCCAC-3') containing BamH I and Hind III cleavage sites and inserted into pCMV-Tag 5 vector (Stratagene) cut with BamH I and Hind III. To construct the expression 


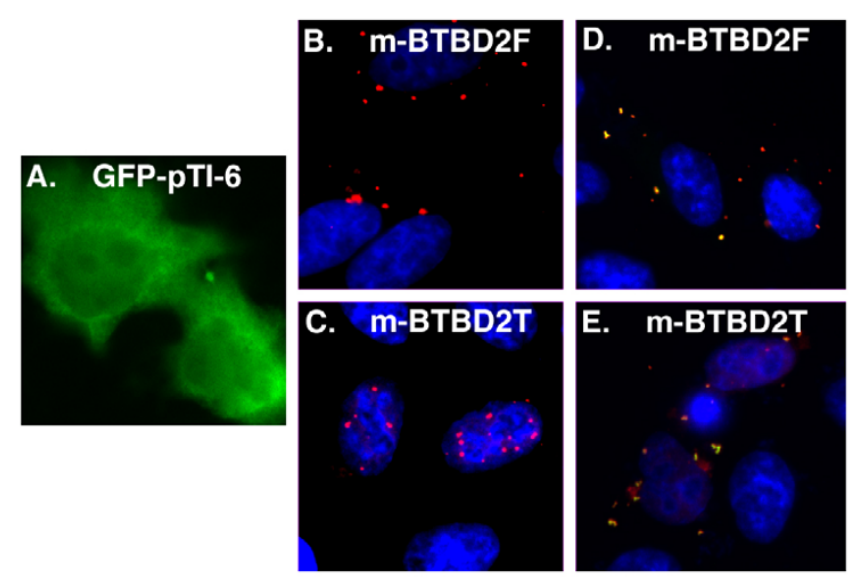

Figure 6

Localization of BTBD2 and co-localization with TOPI. HeLa cells were transfected with: A, GFP-tagged TOPI lacking a nuclear localization (GFP-pTI-6); B, full length BTBD2 containing a C-terminal myc tag (m-BTBD2F); C, truncated BTBD2 having the $N$-terminal half the BTB domain deleted (m-BTBD2T); D, GFP-pTI-6 and m-BTBD2F; and E, GFPPTI-6 and m-BTBD2T.

plasmid encoding C-terminal-myc-tagged truncated BTBD2 having the N-terminal half the BTB domain deleted (m-BTBD2T), the coding region of BTBD2 from the second ATG to almost the end of the 3'-UTR (445-2542 bp) was amplified with primers (Upper primer: 5'-ATGTTCAACGGGGGAATGGCCACAACATC-3', Lower primer: 5'-GGGGAGGACGCAGGTTCCAGGAGT-3') containing BamH I and Hind III cleavage sites and inserted into pCMV-Tag 3 vector (Stratagene) cut with BamH I and Hind III.

\section{Two hybrid screens}

pGBD-cTOP1 was co-transfected with the HeLa cell library (Clontech, Cat. no. HL4000AA) into strain PJ69-4A (MATa, trp 1-190 leu2-3, 112 ura3-52 his3-200 gal4 galso LYS2::GAL1-HIS3 GAL2-ADE2 met2::GAL7-lacZ)[41]. Strong expression of full length GALDB-TOP1 in PJ69-4A was verified by Western blotting with antibodies to both TOP1 and GALDB (not shown). Additionally, the ProQuest two-hybrid system was used because the low copy CEN plasmids may decrease the probability of obtaining weakly interacting false positives that could result from high concentrations of the two-hybrid proteins. In the ProQuest screens, pDBleu-TOP1c was co-transfected with a HeLa cell cDNA library (Life Technologies Cat. No. 11287-018) according the manufacturer protocols. Clones that tested positive in the two-hybrid assays were sequenced across the GAL4AD-prey junction into the coding regions of the prey inserts. Sequences were grouped into cDNA contigs using the SeqMan II program of DNASTAR (LASERGENE).
C-terminal deletions for mapping the interactions using two-hybrid assays were constructed using the exonuclease III/mung bean nuclease method (C-terminal deletions, Exonuclease III/Mung Bean Nuclease Deletion kit, Cat\#460, NEW ENGLAND BioLabs company). N-terminal deletions, in addition to the series obtained through two-hybrid screening, were constructed by amplifying the two-hybrid cDNA clones using primers containing Sma I and EcoR I at the $\mathrm{N}$ - and C-terminus, respectively, and inserting them into both pPC86 and pGEX-2TK vectors. The sense primer sequences are as follows: BTBD1: U1 (395482aa) 5'-GACCCCGGGGAATGATACCGGCTTTAGTTGTGA-3', U2 (342-482aa) 5'-GACCCCGGGCTGGGGTTACAGTGGGACGAGT-3', U3 (213-482aa) 5'GACCCCGGGAAGTGCAGAAGGGTTTACT-3', U4 (303482aa) 5'-GACCCCGGGAAACCTCTTTCTTCATTTTACTGT-3', The same antisense primer was used for all amplifications: $5^{\prime}-$ CGGAATTCTTTGTGGCCATTTATGTTTTTGAC-3'. The sense primers for amplification of truncated BTBD2 fragments were: U1 (504-525aa) 5'-GACCCCGGGCTACGCGGCCGGGAACAACAATG-3', U2 (460525aa) 5'-GACCCCGGGGGTGCTGCCCAACGTCAACTACAC-3', U3 (404-525aa) 5'-GACCCCGGGCGTGGTGGGATTTGGGCTGTATGG-3', U4 (289-525aa) 5'GACCCCGGGCCGCTGGTCCGAGGCCGAGTGTC-3'.

The same antisense primer was used for all amplifications: 5'-CGGAATTCAACCCCGTCCTGATGCTGAGAAAG-3'

\section{Northern blots}

Multiple tissue Northern blots containing $2 \mu \mathrm{g}$ poly (A) + RNA (Human Multiple Tissue Northern Blot IV and Human Multiple Tissue Northern Blot; Catalog numbers: 7766-1 and 7760-1; CLONTECH) were hybridized with 32P-labeled human cDNA probes for TOP1(nucleotides 212-1661, GenBank accession number J03250), BTBD1 (nucleotides 84-3196, GenBank accession number AF355402) or BTBD2 (nucleotides 1229-2668, GenBank accession number AF355797) in Rapid-hyb buffers (Amersham Life Science, RPN1635). Hybridization and washing was carried out according to the manufacturer instructions.

\section{Isolation of the BTBD2 coding region}

The longest BTBD2 cDNA obtained from two-hybrid screening (2413 bp) contained an ORF starting at the 5' end. To obtain the full coding region, four independent cDNA libraries were screened using a nested PCR strategy with upper primers complementary to the vectors and two nested lower primers complementary to BTBD2 (5'TCGTCCGAGTAGAGAAAC-3' and 5'-TCCACGTCGGGCAGCTCAATCTCC-3'). 5'-RACE (Life Technologies) was also used to try to identify longer clones using several different lower primers. Reverse transcription was also performed at $70^{\circ} \mathrm{C}$ using the thermophilic Tth polymerase 
with magnesium as the cofactor to enable it to use mRNA as a template. None of these strategies was successful.

To determine whether the mRNA contained the BTBD2 gene sequences directly upstream of the longest cDNA clone, RT-PCR was used with a sense primer complementary to the predicted start codon. The primers for reverse transcription were complementary to exon 2 (LO and LI, see below). PolyA+ RNA was reverse transcribed using Tth polymerase (Promega) at $70^{\circ} \mathrm{C}$ for $20 \mathrm{~min}$. The resulting products were amplified using nested PCR with the Advantage-GC 2 PCR kit (CLONTECH, Cat\# K-1913-Y) and two 30 cycle rounds with each cycle having two steps (denaturation $94^{\circ} \mathrm{C}, 30 \mathrm{sec}$; annealing and extension $68^{\circ} \mathrm{C}, 3$ min; UO: 5'-ATGGCGGCGGGTGGGAGCGGCGGGCGTGCGTCGTG-3'; UI: 5'-CGGGCCCCGGGACAGACG-3'; LO: 5'-CGGGCAGCTCAATCTCCGTGGATG-3'; LI: 5'-TTGTGGCCATTCCCCCGTTGAACA-3'). To amplify the 306 bp fragment, two rounds of PCR were used: the first round used the LO and UO primers, and the second round used the LO and UI. To amplify the 460 bp fragment, two rounds of PCR were also used, the LO and UO in the first round, and the LI and the UO in the second round.

\section{GST Pull Down Assay}

E. coli containing plasmids encoding GST-BTBD1 or GSTBTBD2 were grown to an O.D. of 0.3 at $25^{\circ} \mathrm{C}$, and $2 \%$ $\mathrm{EtOH}$ was added just prior to induction with $0.1 \mathrm{mM}$ IPTG for 8 hrs. E. coli extracts were prepared using a French Pressure Cell Press (American Instrument Company, Silver Spring, MD). For the GST-pull-down assay $5 \mu \mathrm{g}$ of GST or the GST-fusion proteins were mixed with $150 \mu \mathrm{l} \mathrm{HeLa}$ cell nuclear extracts $\left(5 \times 10^{6}\right.$ cells $\left./ \mathrm{ml}\right)$ and incubated for 2 hr at $4^{\circ} \mathrm{C}(0.25 \mathrm{M} \mathrm{NaCl})$. The bead pellets were then washed 3 times with ice cold PBS before solubilization in SDS-PAGE sample buffer.

\section{TOPI assays}

\section{Supercoil relaxation assay}

HeLa nuclear extract ( $5 \mathrm{ng}$ ) in reaction buffer $(100 \mathrm{mM}$ Tris. $\mathrm{HCl}, \mathrm{pH} 7.5,50 \mathrm{mM} \mathrm{KCl}, 5 \mathrm{mM} \mathrm{MgCl}_{2}, 0.1 \mathrm{mM}$ EDTA, $15 \mu \mathrm{g} / \mathrm{ml} \mathrm{BSA)} \mathrm{was} \mathrm{incubated} \mathrm{with} 5 \mu \mathrm{g}$ of purified GST or GST-BTBD2 on ice for 15 min before the addition of $0.25 \mu \mathrm{g}$ plasmid and incubation at $30^{\circ} \mathrm{C}$ for $0-24 \mathrm{~min}$. Reactions were stopped by adding SDS (1\% final) and proteinase $\mathrm{K}(0.5 \mathrm{mg} /$ reaction $)$.

\section{DNA cleavage assays}

Purified TOP1 was pre-incubated with GST or GST-BTBD2 for 20 min before addition of the DNA substrate, which was either an end-labeled 36 bp fragment containing a single strong TOP1 cleavage site [24], or a 161 bp PvuIIHindIII fragment of pBluescript (Stratagene) [25]). End labeling of the DNA substrates was as previously de- scribed [24,25]. Cleavage reactions were continued for 20 min before stopping with $0.5 \%$ SDS.

\section{Abbreviations}

aa, amino acid; BTB: Broad-Complex, Tramtrack and Brica-brac; GST, glutathione-S-transferase; NLS, nuclear localization signal; ORF, open reading frame; RT-PCR, reverse transcription - polymerase chain reaction; TOP1, DNA topoisomerase I.

\section{Acknowledgements}

We thank Dr's Yin-Yuan Mo and Bill Beck for providing the GFP-TOPI expression plasmids and Dr. Todd Martensen for critical comments on the manuscript.

\section{References}

I. Wang Z, Roeder RG: DNA topoisomerase I and PC4 can interact with human TFIIIC to promote both accurate termination and transcription reinitiation by RNA polymerase III. Mol Cell 1998, I:749-757

2. Merino A, Madden K, Lane WS, Champoux J, Reinberg D: DNA topoisomerase $\mathrm{I}$ is involved in both repression and activation of transcription. Nature 1993, 365:227-232

3. Gobert C, Skladanowski A, Larsen AK: The interaction between p53 and DNA topoisomerase $I$ is regulated differently in cells with wild-type and mutant p53. Proc Natl Acad Sci USA 1999, 96: $10355-10360$

4. Haluska P Jr, Saleem A, Edwards TK, Rubin EH: Interaction between the $\mathbf{N}$-terminus of human topoisomerase I and SV40 large T antigen. Nucleic Acids Res 1998, 26:184I-I847

5. Edwards TK, Saleem A, Shaman JA, Dennis T, Gerigk C, Oliveros E, Gartenberg MR, Rubin EH: Role for Nucleolin/Nsrl in the Cellular Localization of Topoisomerase I. J Biol Chem 2000, 275:36|8I-36|88

6. Haluska P Jr, Saleem A, Rasheed Z, Ahmed F, Su EW, Liu LF, Rubin EH: Interaction between human topoisomerase $I$ and a novel RING finger/arginine-serine protein. Nucleic Acids Res 1999, 27:2538-2544

7. Straub T, Grue P, Uhse A, Lisby M, Knudsen BR, Tange TO, Westergaard O, Boege F: The RNA-splicing factor PSF/p54 controls DNA-topoisomerase I activity by a direct interaction. J Biol Chem 1998, 273:26261-26264

8. Labourier E, Rossi F, Gallouzi IE, Allemand E, Divita G, Tazi J: Interaction between the $\mathrm{N}$-terminal domain of human DNA topoisomerase $I$ and the arginine-serine domain of its substrate determines phosphorylation of SF2/ASF splicing factor. Nucleic Acids Res 1998, 26:2955-2962

9. Karayan L, Riou JF, Seite P, Migeon J, Cantereau A, Larsen CJ: Human ARF protein interacts with Topoisomerase I and stimulates its activity. Oncogene 200I, 19:836-848

10. Shaiu WL, Hsieh TS: Targeting to transcriptionally active loci by the hydrophilic $\mathrm{N}$-terminal domain of Drosophila DNA topoisomerase I. Mol Cell Biol 1998, 18:4358-4367

II. Shaiu WL, Hu T, Hsieh TS: The hydrophilic, protease-sensitive terminal domains of eucaryotic DNA topoisomerases have essential intracellular functions. Pac Symp Biocomput 1999578589

12. Suzuki T, Uchida-Toita M, Andoh T, Yoshida M: HTLV-I tax oncoprotein binds to DNA topoisomerase $I$ and inhibits its catalytic activity. Virology 2000, 270:29I-298

13. Desai SD, Li TK, Rodriguez-Bauman A, Rubin EH, Liu LF: Ubiquitin/ $26 \mathrm{~S}$ proteasome-mediated degradation of topoisomerase I as a resistance mechanism to camptothecin in tumor cells. Cancer Res 200I, 61:5926-5932

14. Mao Y, Sun M, Desai SD, Liu LF: A possible repair response to topoisomerase-mediated DNA damage. Proc Natl Acad Sci USA 2000, 97:4046-405।

15. Staron K, Samuels DS: Phosphorylation of serine residues in the $\mathrm{N}$-terminal domains of eukaryotic type I topoisomerases. Mol Biol Rep 1998, 25:|57-|6| 
16. Desai SD, Liu LF, Vazquez-Abad D, D'Arpa P: Ubiquitin-dependent destruction of topoisomerase $I$ is stimulated by the antitumor drug camptothecin. J Biol Chem 1997, 272:24I59-24I64

17. Staron K, Kowalska-Loth B, Zabek J, Czerwinski RM, Nieznanski K, Szumiel I: Topoisomerase $I$ is differently phosphorylated in two sublines of L5 I 78Y mouse lymphoma cells. Biochim Biophys Acta 1995, 1 260:35-42

18. Stewart L, Ireton GC, Champoux JJ: The domain organization of human topoisomerase I. J Biol Chem 1996, 27I:7602-7608

19. Stewart L, Ireton GC, Parker LH, Madden KR, Champoux JJ: Biochemical and biophysical analyses of recombinant forms of human topoisomerase I. J Biol Chem 1996, 27 I:7593-760I

20. Stewart L, Ireton GC, Champoux J): Reconstitution of human topoisomerase I by fragment complementation. J Mol Biol 1997, 269:355-372

21. Carim-Todd L, Sumoy L, Andreu N, Estivill X, Escarceller M: Identification and characterization of BTBDI, a novel BTB domain containing gene on human chromosome I5q24. Gene 200I, 262:275-281

22. Pruitt KD, Maglott DR: RefSeq and LocusLink: NCBI gene-centered resources. Nucleic Acids Res 200 I, 29: I37-I40

23. Lash AE, Tolstoshev CM, Wagner L, Schuler GD, Strausberg RL, Riggins G], Altschul SF: SAGEmap: a public gene expression resource. Genome Res 2000, 10:1051-1060

24. Pourquier P, Takebayashi $Y$, Urasaki Y, Gioffre C, Kohlhagen G, Pommier $Y$ : Induction of topoisomerase I cleavage complexes by I-beta-D-arabinofuranosylcytosine (ara-C) in vitro and in ara-C-treated cells. Proc Natl Acad Sci USA 2000, 97:I 1885-I890

25. Pommier Y, Kohlhagen G, Wu C, Simmons DT: Mammalian DNA topoisomerase I activity and poisoning by camptothecin are inhibited by simian virus $\mathbf{4 0}$ large T antigen. Biochemistry 1998, 37:3818-3823

26. Muller MT, Pfund WP, Mehta VB, Trask DK: Eukaryotic type I topoisomerase is enriched in the nucleolus and catalytically active on ribosomal DNA. Embo ] 1985, 4: I 237-I243

27. Danks MK, Garrett KE, Marion RC, Whipple DO: Subcellular redistribution of DNA topoisomerase I in anaplastic astrocytoma cells treated with topotecan. Cancer Res 1996, 56:1664-1673

28. Wadkins RM, Danks MK, Horowitz L, Baker SD: Characterization of topotecan-mediated redistribution of DNA topoisomerase I by digital imaging microscopy. Exp Cell Res 1998, 24 I:332339

29. Zini N, Santi S, Ognibene A, Bavelloni A, Neri LM, Valmori A, Mariani E, Negri C, Astaldi-Ricotti GC, Maraldi NM: Discrete localization of different DNA topoisomerases in HeLa and $\mathrm{K} 562$ cell nuclei and subnuclear fractions. Exp Cell Res 1994, 21 0:336-348

30. Mo YY, Wang P, Beck WT: Functional expression of human DNA topoisomerase I and its subcellular localization in HeLa cells. Exp Cell Res 2000, 256:480-490

31. Redinbo MR, Stewart L, Kuhn P, Champoux J], Hol WG: Crystal structures of human topoisomerase $I$ in covalent and noncovalent complexes with DNA. Science 1998, 279:1504-1513

32. Yudate HT, et al: HUNT: launch of a full-length cDNA database from the Helix Research Institute. Nucleic Acids Res 200I, 29:185-188

33. Ahmad KF, Engel CK, Prive GG: Crystal structure of the BTB domain from PLZF. Proc Natl Acad Sci USA 1998, 95:12123-12I 28

34. Li X, Peng H, Schultz DC, Lopez-Guisa JM, Rauscher FJ 3rd, Marmorstein R: Structure-function studies of the BTB/POZ transcriptional repression domain from the promyelocytic leukemia zinc finger oncoprotein. Cancer Res 1999, 59:52755282

35. Adams J, Kelso R, Cooley L: The kelch repeat superfamily of proteins: propellers of cell function. Trends Cell Biol 2000, I0:1724

36. Lai F, Orelli BJ, Till BG, Godley LA, Fernald AA, Pamintuan L, Le Beau MM: Molecular characterization of KLHL3, a human homologue of the Drosophila kelch gene. Genomics 2000, 66:65-75

37. Bomont $P$, Cavalier L, Blondeau F, Hamida CB, Belal S, Tazir M, Demir E, Topaloglu H, Korinthenberg R, Tuysuz B, Landrieu P, Hentati F, Koenig $M$ : The gene encoding gigaxonin, a new member of the cytoskeletal BTB/kelch repeat family, is mutated in giant axonal neuropathy. Nat Genet 2000, 26:370-374

38. Carim-Todd L, Escarceller M, Estivill X, Sumoy L: Cloning of the novel gene TM6SF I reveals conservation of clusters of paral- ogous genes between human chromosomes 15q24 $\rightarrow$ q26 and I9pI3.3 $\rightarrow$ p I 2. Cytogenet Cell Genet 2000, 90:255-260

39. Dynan WS: Understanding the molecular mechanism by which methylation influences gene expression. Trends Genet 1989, 5:35-36

40. Nakamura Y, Miura K, Fujino Y, Iwao H, Ogita S, Yamanaka S: Evolution, structure, and expression of GNPI/Oscillin orthologous genes. Genomics 2000, 68: $179-186$

41. James P, Halladay J, Craig EA: Genomic libraries and a host strain designed for highly efficient two-hybrid selection in yeast. Genetics 1996, 144:1425-1436
Publish with BioMed Central and every scientist can read your work free of charge

"BioMedcentral will be the most significant development for disseminating the results of biomedical research in our lifetime." Paul Nurse, Director-General, Imperial Cancer Research Fund

Publish with BMC and your research papers will be:

- available free of charge to the entire biomedical community

- peer reviewed and published immediately upon acceptance

- cited in PubMed and archived on PubMed Central

- yours - you keep the copyright

Submit your manuscript here:

http://www.biomedcentral.com/manuscript/
BioMedcentral.com ditorial@biomedcentral.com 